Editorial

\title{
Ambulatory Blood Pressure Monitoring in Pregnancy: Simple and Effective
}

\author{
Amrutha Kakollu',๑ \\ ${ }^{1}$ Department of Obstetrics and Gynecology, Amrutha Clinic, \\ Hyderabad, Telangana, India \\ Ind J Car Dis Wom 2021;6:25-26.
}

The current era may be considered as the finest in the progress of science and technology. Considering the extensive armamentarium at our disposal, it is nothing less than overwhelming while deciding on the best choice for our patients. The joy of motherhood is delightful for all, and yet for some women, the journey has its own perils. The role of the obstetrician is of utmost importance in aiding our pregnant women to achieve a healthy and happy outcome. This mandates the use of simple measures to a complex battery of investigations and treatments as needed.

Hypertensive disorders of pregnancy comprise an important aspect of antenatal care; hence, predicting accurate identification and well-timed treatment is essential at any gestational age. Preeclampsia and eclampsia are a significant cause for maternal-fetal morbidity and mortality. Numerous methods have been recommended for predicting and screening antenatal women from the first trimester for preeclampsia with variable accuracy. Women with previous history of preeclampsia, and preexisting chronic hypertension is at significantly high risk for developing preeclampsia. ${ }^{1}$ Frequently used markers at 11 to 13 weeks of gestation along with clinical risk stratification are mean arterial pressure, uterine artery pulsatility index, serum pregnancy-associated plasma protein-A, and serum placental growth factor. ${ }^{2}$

The earliest and easiest method of identifying women at risk of developing hypertension is by the measurement of blood pressure (BP) during antenatal visit. It has been reported that women with increased BP recordings in their first trimester are more likely to develop preeclampsia., ${ }^{2,3}$

Recording of BP in pregnant women should be done meticulously as high recordings cannot be ignored. Establishing a proper protocol for BP measurement and training the staff is important and can help to minimize false positive measurements in the outpatient department (OPD). The use of mercury sphygmomanometer was traditionally considered as the gold standard. National Institute for Health and Care excellence recommends the usage of appropriate cuff size,

Address for correspondence Amrutha Kakollu, MS, FMAS, Amrutha Clinic Sri Nagar Colony, Hyderabad, Telangana, 500073, India (e-mail:amrutha9jaideep@gmail.com).
DOI https://doi.org/ 10.1055/s-0041-1726611

published online April 9, 2021 initial cuff inflation to 20 to $30 \mathrm{~mm}$ above the palpable systolic pressure, deflating the cuff at the rate of $2 \mathrm{mmHg}$ per second, and Korotkoff phase $\mathrm{V}$ for diastolic BP to improve accuracy. ${ }^{4}$

BP recording by auscultatory or automated devices is subjective. During manual measurement, deflating the cuff quickly can lead to under-recording of systolic BP and over recording of diastolic BP. In case of automated machines, they need to be regularly calibrated. A single high BP recording is insufficient to predict or diagnose hypertension. In an individual, BP may be influenced by various emotional and physical aspects.

Dr. Maurice Sokolow in the 1950s was the first to investigate if a single BP recording in the clinic is illustrative of the BP throughout the day and proposed methods of recording ambulatory BP. ${ }^{5}$ However, this was clinically validated a decade later, and the automatic noninvasive monitor was invented in the 1970 . The usage of ambulatory blood pressure monitoring (ABPM) has been explored in various circumstances. The study done by Halligan et al in 1993 titled "24-hour ABPM in a primigravid population" was the first of its kind to establish the normal circadian changes in BP throughout the day in pregnant women. ${ }^{6}$ They identified that lowest diastolic BP (diurnal and nocturnal) was recorded between $18 \pm 24$ and increased at $33 \pm 40$ weeks in the same manner as the systolic blood pressure. In their study, the ABPM (both systolic and diastolic) during the day were significantly lower than the clinic BP recordings up to 33 weeks. After 33 weeks, they noted no difference between clinic and ABPM readings. This study was monumental in documenting the physiological variation of BP in pregnancy and the midtrimester fall of BP that then rises in the third trimester and plateaus.

The subsequent studies have tried to use ABPM as a predictor for preeclampsia. The study by Oxford in 1993 stated that women who developed preeclampsia in their study had significantly higher systolic and mean arterial pressures 
at 18 and 28 weeks of gestation and diastolic pressure at 28 weeks. ${ }^{7}$ The drawback of this study was the low predictive value and the intensive monitoring of normal women that can lead to increased economic burden and emotional anxiety to the pregnant women as well. Further studies were conducted with similar results. ${ }^{8}$ Though the higher recordings were noted in women who consequently developed preeclampsia, there was significant overlay with normal women. These findings are in concordance with the findings of this study where antenatal women with increased nocturnal diastolic pressure with diurnal maximum systolic blood pressures were noted as the best predictors of maternofetal outcome.

Shannan et al in 1998 conducted a study recruiting 109 primigravid women beyond 20 weeks of gestation and high $\mathrm{BP}$ recording in the OPD who were monitored with ABPM from 9 A.M. to 11 P.M. ${ }^{5}$ The primary outcome in this study was the onset of proteinuria, delivery by cesarean section, preterm delivery, low birthweight, and neonatal intensive care. In this study, ABPM was a better predictor of relative risk for all the outcomes except need of cesarean delivery in comparison with clinic BP recordings. This study emphasizes that ABPM has a role in accurately diagnosing hypertension in women who otherwise maybe falsely diagnosed as hypertensive. Also, decreasing the threshold from 140/90 to $130 / 85 \mathrm{mmHg}$ was seen to improve sensitivity but decreased specificity for all outcomes. These findings were confirmed in a subsequent study by the same group in 300 pregnant women where ABPM had a role in predicting severe hypertension, but not proteinuria. ${ }^{9}$ In the present study, women who had no nocturnal reduction of systolic and diastolic blood pressure were at increased risk of adverse maternofetal events. Therefore, ABPM has a role in identifying women who do not have the nocturnal dip in blood pressure and monitoring them frequently, anticipate complications with earlier interventions.

ABPM in high-risk pregnancy can provide aid in early risk assessment and can be used as a guide for establishing prophylactic or therapeutic intervention, identifying women who may develop fetal growth restriction and need preterm delivery. ${ }^{10}$ In this study, the most common antenatal complication was intrauterine growth restriction. ABPM can help to identify women who are at a higher risk for complications and an opportunity for earlier intervention.

Kasim et al in 2019 have conducted a study on 47 low-risk pregnant women to evaluate if ABPM is better than sphygmomanometer and concluded that ABPM was accurate with advantage of assessing BP variation in a better way. ${ }^{11} \mathrm{ABPM}$ is considered as the ideal way to diagnose white coat hypertension. In the study by Sood et al, $48.15 \%$ patients who were diagnosed with gestational hypertension in the OPD were later identified as white coat hypertension with the aid of using ABPM. ${ }^{12}$

However, the limitation of cost and the physical discomfort of carrying the device need to be considered in patients who may need repeated evaluation for a longer period of time. ABPM may have a role in monitoring high-risk patients for earlier clinical intervention that may reduce the need of intensive therapy during the further course of pregnancy.

\section{Conclusion}

Preeclampsia is a multifactorial entity with several etiological processes. The most common reason being inadequate placentation that does not allow the physiological adaptation in pregnancy and results in high BP recordings and endothelial damage that are responsible for the various possible outcomes of the condition. Quoting Albert Einstein "everything should be made as simple as possible, but not simpler." The idea of being able to identify women who will develop preeclampsia by means of noninvasive ABPM may sound compelling. However, this is to be taken with a pinch of salt and combining clinical risk assessment and biomarkers, ultrasonography with ABPM will definitely improve the predictive outcome of maternofetal complications. Studies with significant sample size are needed to validate the same.

\section{Conflict of Interest}

None declared.

\section{References}

1 Bartsch E, Medcalf KE, Park AL, Ray JG; High Risk of Preeclampsia Identification Group. Clinical risk factors for pre-eclampsia determined in early pregnancy: systematic review and meta-analysis of large cohort studies. BMJ 2016; 353:i1753

2 Poon LC, Sahota D. Screening and prevention of preeclampsia. Maternal-Fetal Medicine 2019;1(1):25-30

3 Poon LC, Kametas NA, Valencia C, Chelemen T, Nicolaides KH. Hypertensive disorders in pregnancy: screening by systolic diastolic and mean arterial pressure at 11-13 weeks. Hypertens Pregnancy 2011;30(1):93-107

4 National Institute for Health and Care Excellence. Antenatal Care. NICE Clinical Guideline 62. London: NICE; 2008

5 Shennan AH, Halligan AW. Ambulatory blood pressure monitoring in pregnancy. Fetal Matern Med Rev 1998;10(2):69-89

6 Halligan A, O'Brien E, O'Malley K, et al. Twenty-four-hour ambulatory blood pressure measurement in a primigravid population. J Hypertens 1993;11(8):869-873

7 Kyle PM, Clark SJ, Buckley D, et al. Second trimester ambulatory blood pressure in nulliparous pregnancy: a useful screening test for pre-eclampsia? Br J Obstet Gynaecol 1993; 100(10):914-919

8 Higgins JR, Walshe JJ, Halligan A, O’Brien E, Conroy R, Darling MR. Can 24-hour ambulatory blood pressure measurement predict the development of hypertension in primigravidae? Br J Obstet Gynaecol 1997;104(3):356-362

9 Penny JA, Halligan AW, Shennan AH, et al. Automated, ambulatory, or conventional blood pressure measurement in pregnancy: which is the better predictor of severe hypertension? Am J Obstet Gynecol 1998;178(3):521-526

10 Ayala DE, Ucieda R, Hermida RC. Chronotherapy with low-dose aspirin for prevention of complications in pregnancy. Chronobiol Int 2012b;29In press

11 Kasim HH, Masri MA, Noh NA, Mokhtar A, Mokhtar RH. Is ABPM a better device for monitoring pregnancy than sphygmomanometer? Int J Cardiol 2019;297:25-26

12 Anne SS, Ashta KK, Kumar R. Comparison of ambulatory blood pressure monitoring and self-blood pressure monitoring for diagnosing white coat hypertension amongst pregnant women. Int J Reprod Contracept Obstet Gynecol 9(1):275 